\title{
SOME MATHEMATICAL CONCEPTS IN GEOMETRY OF MASSES
}

\author{
GORDANA JELIĆ ${ }^{1, \star}$, DEJAN STOŠOVIĆ ${ }^{1}$ \\ ${ }^{1}$ Faculty of Technical Sciences, University in Priština, Kosovska Mitrovica, Serbia
}

\begin{abstract}
The study of the distribution of geometrical points, loaded by some scalars plays an important role in various fields of science, of theoretical and practical character. Since such study was first applied and studied when mass had the role of played a scalar, the mass loaded point was named material point, and the discipline dealing with the arrangement of material points in space is called the geometric mass. In that discipline, in the general case under mass one should imply a scalar of arbitrary nature, which can be negative as well. For example, the discipline includes the study of distribution of magnetic or electrical masses, which can be positive and negative. This paper presents some concepts from the geometry of masses that play an important role, particularly in mechanics and physics.
\end{abstract}

Keywords: Material point, Geometry of masses, Body density, Linear density, Surface density, Homogeneous material, Non-homogeneous materials, Center of mass

\section{INTRODUCTION}

The set of material points in the final or infinite number is called the material system. The material points of system are arranged discretely or continuously. Each mass, even the smallest, needs to take up some, perhaps even a very small volume. On the other hand, for a large mass we can take a geometric point and consider that the point with the overall mass represents the mass (Awrejcewicz, 2012). If it can be prove that all the points of the entire mass move as the selected representative point, then selected geometric point represents the entire mass and exists as a discrete material point (Mirtich, 1996; Whitaker, 1992).

\section{THE CONCEPT OF DENSITY OF CONTINUOUSLY DIS- TRIBUTED MASSES}

For the system of continuously distributed masses firstly is introduced the concept of medium volumetric density, as a ratio $\Delta m / \Delta V$ where $\Delta V$ is the volume and $\Delta m$ is the mass in the volume. Then, it can be introduced the concept of density of a given body at a given point $M$ as

$$
\sigma=\lim _{\Delta V \rightarrow 0} \frac{\Delta m}{\Delta V}=\frac{\mathrm{d} m}{\mathrm{~d} V}
$$

where $\mathrm{d} m$ is the mass differential and $\mathrm{d} V$ is the differential of the corresponding volume. In general case, the density $\sigma$ depends on the position of the point $M$ in the given body. It is expressed by the following equation $\sigma=f(M)=f(\mu)$, where $\mu$ is the radius-vector of point $M$ with respect to a origin, i.e. of permanent position $O$ to the body, that is $\mu=\overrightarrow{O M}$. If the vector $\mu$ is determined using the Cartesian coordinates $(x, y, z)$ of the point $M$, then the density is scalar function $\sigma=f(x, y, z)$. Finally, if the density is given for all points of volume $V$, the mass of the body $m$ is determined by the integral (Bilimović, 1961; Dorogovtsev, 1987)(in english):

$$
m=\iiint_{V} \sigma \mathrm{d} V=\iiint_{V} \sigma(x, y, z) \mathrm{d} x \mathrm{~d} y \mathrm{~d} z
$$

extended to the entire volume $V$ of the body.

Regardless of each mass having have to occupy a volume, it can also be discussed on the surface and the linear distribution of the masses. The examples of surface mass distribution can be material plates, covers, shells, sheet metal, etc. and the examples for linear distribution of masses can be rods, wires, cords etc. Related to such distributions we can talk about medium surface density $\Delta m / \Delta A$, where $\Delta m$ is the mass and $\Delta A$ is a part of the surface of the mass. Limit value

$$
\sigma_{1}=\lim _{\Delta A \rightarrow 0} \frac{\Delta m}{\Delta A}=\frac{\mathrm{d} m}{\mathrm{~d} A},
$$

is surface density in the given point $M$. Similarly,

$$
\sigma_{2}=\lim _{\Delta \ell \rightarrow 0} \frac{\Delta m}{\Delta \ell}=\frac{\mathrm{d} m}{\mathrm{~d} \ell},
$$

is the linear density (of a given wire at a given point $M$ ).

Note that the above densities have different dimensions, i.e. it is valid

$$
[\sigma]=M L^{-3}, \quad\left[\sigma_{1}\right]=M L^{-2}, \quad\left[\sigma_{2}\right]=M L^{-1}
$$

where $M$ is the mass, as measured by, say, grams, and $L$ indicates the length in centimeters. Determination of body mass of density is calculated as the double integral

$$
m=\iint_{A} \sigma_{1}(u, v) \sqrt{E G-F^{2}} \mathrm{~d} u \mathrm{~d} v=\iint_{A} \sigma_{1}(x, y) \mathrm{d} x \mathrm{~d} y,
$$

where $A$ is the surface of the body. In the case of linear mass distribution we have the following integral

$$
m=\iint_{L} \sigma_{2}(\ell) \mathrm{d} \ell
$$


where $\mathrm{d} \ell$ is the element of length extended to the entire length of the curve. For a real wire it is

$$
m=\iint_{L} \sigma_{2}(x) \mathrm{d} x
$$

If $\sigma, \sigma_{1}, \sigma_{2}$ are constant values, the matter of the body is homogeneous and to calculate body mass it is sufficient to know the volume i.e. the surface or the length of the body, because in these cases

$$
m=\sigma V=\sigma_{1} A=\sigma_{2} L .
$$

In the cases of non-homogeneous (heterogeneous) matter, body mass is not proportional to the density.

\section{CENTER OF MASSES OR CENTER OF INERTIA}

If related to the point $M$ is the mass $m$ and $\overrightarrow{A M}$ is the vector of position of the point $M$ in respect to point $A$, the result $m \overrightarrow{A M}$ is called the position vector burdened by mass $m$ (of the point $M$ in respect to point $A)($ Bessonov \& Song, 2001). Suppose that we have a set of $n$ points of $M_{1}, M_{2}, \ldots, M_{n}$ with masses $m_{1}, m_{2}, \ldots, m_{n}$ and one particular point of space $A$. The position vector for each point of this set may be constructed burdened by mass relative to point $A$ and we can make a summation of all constructed vectors $m_{1} \overrightarrow{A M_{1}}+\cdots+m_{n} \overrightarrow{A M_{n}}$. The result of this sum is a vector starting at point $A$. If this vector is divided by the entire mass $m=\sum_{j=1}^{n} m_{j}$, we will get a new vector, whose the end we will mark with $C$. In this manner, the vector equation

$$
m \overrightarrow{A C}=\sum_{j=1}^{n} m_{j} \overrightarrow{A M}_{j}
$$

determines the point $C$. Thus defined point $C$ is called the center (of inertia of the masses).

In following, we shall examine some typical properties of the center of inertia. In this sense, we shall prove two simple propositions that justify the meaning that this point was called the center.

Theorem 1. The position of point $C$ of a given material system do not depends on the choice of point $A$, i.e. the beginning of the position vector of all constructed points.

Proof. Let us take as the starting point, besides $A$, some other an arbitrary point $A_{1} \neq A$. If we denote with $C_{1}$ the appropriate center of inertia of the point $A_{1}$, we obtain the new vector equation

$$
m \overrightarrow{A C_{1}}=\sum_{j=1}^{n} m_{j} \overrightarrow{A_{1} M_{j}}
$$

Notice that $\overrightarrow{A C_{1}}=\overrightarrow{A_{1} A}+\overrightarrow{A C}+\overrightarrow{C C_{1}}$ and $\overrightarrow{A_{1} M_{j}}=\overrightarrow{A_{1} A}+\overrightarrow{A M_{j}}$, $j=1, \ldots, n$ (see Fig. 1). Now, if we put those vectors in the Eq.(2), and taking into account the Eq.(1), we get

$m \overrightarrow{A_{1} A}+m \overrightarrow{A C}+m \overrightarrow{C C_{1}}=\sum_{j=1}^{n} m_{j} \overrightarrow{A_{1} A}+\sum_{j=1}^{n} m_{j} \overrightarrow{A M_{j}}$

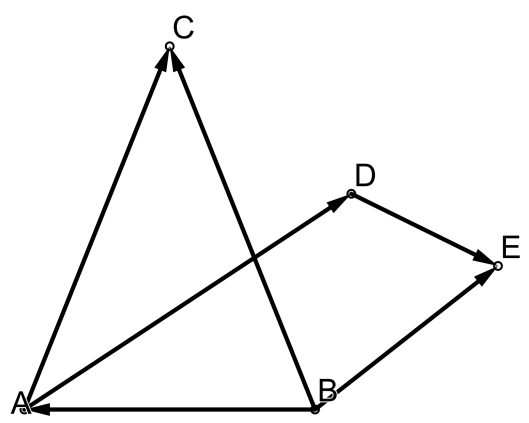

Figure 1. Determining the center (of inertia of the masses).

which implies $\overrightarrow{C C_{1}}=\overrightarrow{0}$. Thus, the center $C_{1}$ coincides with the original one.

Remark 2. According to Theorem 1, it follows that the position of the center depends only on the size of the masses and the distribution of these masses in the area. Therefore, the center of masses is an important natural point of any material system.

Theorem 3. The sum of the position vectors of points $M_{j}$, in relation to the center $C$ and weighted by the masses $m_{j}$ is equal to zero, i.e.

$$
\sum_{j=1}^{n} m_{j} \overrightarrow{C M_{j}}=\overrightarrow{0}
$$

Proof. Let us consider again the Eq.(1). In order to determine the point $C$, into this equation let we put $\overrightarrow{A M}_{j}=\overrightarrow{A C}+\overrightarrow{C M}_{j}, j=$ $1, \ldots, n$. Thus, we have:

$$
m \overrightarrow{A C}=\sum_{j=1}^{n} m_{j}\left(\overrightarrow{A C}+\overrightarrow{C M_{j}}\right)
$$

or, equivalently:

$$
\sum_{j=1}^{n} m_{j} \overrightarrow{C M}_{j}=\overrightarrow{0}
$$

which was supposed to be proven.

Remark 4. From the basic vector Eq.(1) for the center $C$ it follows that:

$$
\overrightarrow{A C}=\sum_{j=1}^{n} \lambda_{j} \overrightarrow{A M}_{j}
$$

where $\lambda_{j}=m_{j} / m, j=1, \ldots, n$ and $\sum_{j=1}^{n} \lambda_{j}=1$. Thus, vector $\overrightarrow{A C}$ is the convex combination of vectors $\overrightarrow{A M_{1}}, \ldots, \overrightarrow{A M_{n}}$. In the Cartesian coordinates, Eq.(4) can be written as:

$$
\overrightarrow{\mu_{c}}=\sum_{j=1}^{n} \lambda_{j} \overrightarrow{\mu_{j}}
$$

where $\vec{\mu}_{c}=\overrightarrow{A C}=\left(x_{c}, y_{c}, z_{c}\right)$ and $\vec{\mu}_{j}=\overrightarrow{A M}_{j}=\left(x_{j}, y_{j}, z_{j}\right)$. In this way, to the vector Eqs.(4)-(5) correspond the following three scalar equations

$$
x_{c}=\sum_{j=1}^{n} \lambda_{j} x_{j}, \quad y_{c}=\sum_{j=1}^{n} \lambda_{j} y_{j}, \quad z_{c}=\sum_{j=1}^{n} \lambda_{j} z_{j} .
$$


These are the basic scalar equations for determining the position of the center of masses.

Remark 5. If the masses of a material system are distributed in a certain area continuously, the sums extended to all material points of the system pass into defined integrals extended to the areas of continuous matter. Then we have the vector equations:

$$
\overrightarrow{A C} \iiint_{V} \mathrm{~d} m=\iiint_{V} \vec{\mu} \mathrm{d} m
$$

or

$$
\overrightarrow{A C} \iiint_{V} \sigma \mathrm{d} V=\iiint_{V} \sigma \vec{\mu} \mathrm{d} m
$$

The scalar form of $O_{x}$ is:

$$
x_{c}=\iiint_{V} \sigma x \mathrm{~d} V: \iiint_{V} \sigma \mathrm{d} V
$$

The analog integrals apply in the case of masses continuously distributed over the surface and along the lines.

Remark 6. If the matter of body is homogeneous, the multiplier of density $\sigma$ entering the numerator and denominator can be shortened, and thus for the homogeneous bodies we have patterns in which the mass is not included. These patterns can be considered as forms for the determination of the center of inertia of a volume i.e. of a surface or line. Thus, we have:

$x_{c}=\iiint_{V} x \mathrm{~d} V: \iiint_{V} \mathrm{~d} V, x_{c}^{\prime}=\iint_{A} x \mathrm{~d} A: \iint_{A} \mathrm{~d} A, x_{c}^{\prime \prime}=\iint_{L} x \mathrm{~d} l: \int_{L} \mathrm{~d} l$

Remark 7. If the arrangement is symmetrical with respect to a plane, or a line or point, the center of masses must lie in that plane, on the line or at that point.

\section{PAPPOS-GULDIN'S THEOREMS}

Regarding the concept of the center of masses we list two so-called Pappos-Guldin's theorems.

Theorem 8. The area which is obtained by rotating the arc of a curve in the plane about an axis in the plane, which does not cut the curve, is equal to the product of the arc length and circumference of a circle described by the mass center of this arc.

Proof.

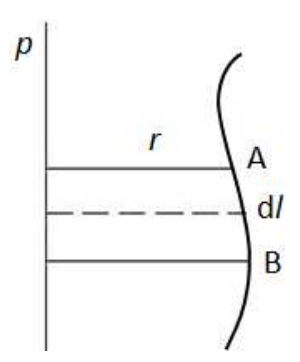

Figure 2. Arc of a curve in the plane.
Let a part of the flat curve, from point $A$ to point $B$, rotate about an axis $p$ on the curve plane and does not cut the curve, but only points $A$ and $B$ can belong to the axis. Let $\Delta l$ be the distance between two very close points of the curve (see Fig. 2). Area formed by $\Delta l$ in reversing (i.e. the side surface of the cylinder, coupe, truncated cone or a circular ring) is equal to $2 \pi r_{s} \Delta l$, where $r_{s}$ is the distance of the middle of the line $\Delta l$ from the axis of rotation. Since in the border case the area is expressed by $2 \pi r \mathrm{~d} l$, where $\mathrm{d} l$ is the differential of the curve arc and $r$ is the distance of an arbitrary point of the arc from the rotation axis, accordingly the surface $S$, described by the $\operatorname{arc} A B$ is equal to:

$$
S=2 \pi \int_{L} r \mathrm{~d} l, \text { where } \mathrm{L} \text { is the arc of } \mathrm{AB} .
$$

If we use $r_{c}$ to mark the distance of the center of mass $C$ of the arc $L$ from the straight line $p$, then we have the equation:

$$
L r_{c}=\int_{L} r \mathrm{~d} l
$$

If this value of integral is put into the previous equation it gives:

$$
S=2 \pi r_{c} L
$$

Theorem 9. The volume which is obtained by rotating the flat surface around the axis in the plane, which does not cut the surface, equals to the product of the surface value and the circumference of a circle described by the center of masses of the surface.

Proof. Suppose that the flat surface $P$ rotates around the axis $p$,

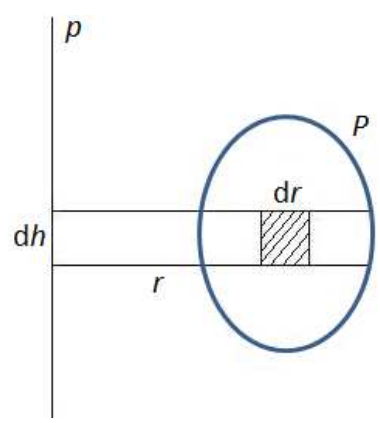

Figure 3. Flat surface in a plane.

which does not cut the contour of the surface (see Fig. 3). Volume $V$, which is obtained by reversing the surface, can be calculated using the double integral:

$$
V=2 \pi \iint_{P} r \mathrm{~d} r \mathrm{~d} h
$$

where $r$ is the distance of the point of elementary rectangle of dimensions $d r$ and $d h$ from the axis of rotation. If we write down the equation for determining the center of mass $C$ of the area $P$ with respect to line $p$, the following is obtained

$$
\operatorname{Pr}_{c}=\iint_{P} r \mathrm{~d} r \mathrm{~d} h
$$


where $r_{c}$ is the distance of the center of mass of the surface $P$ from the line $p$. When this integral value is put into the previous equation, we get:

$$
V=2 \pi r_{c} P
$$

which is supposed to be obtained.

\section{AXIAL SQUARE MOMENT OF INERTIA}

Let there be given the line $p$ and the material point of the mass $m$ at the distance $d$ from the line. The product $m d^{2}$ is called the axial square moment of the given material point relative to the given line. If there are more material points with masses $m_{1}, m_{2}, \ldots, m_{n}$ at distances $d_{1}, d_{2}, \ldots, d_{n}$ of the given line $p$, for the moment of inertia of the system we have the equation and the mark:

$$
I_{p}=\sum_{i=1}^{n} m_{i} \mathrm{~d}_{i}^{2}
$$

If the coordinates of mass $m_{i}$ are marked by $x_{i}, y_{i}, z_{i}$ in relation to the Cartesian coordinate system, then three moments of inertia can be introduced:

$I_{x}=\sum m_{i}\left(y_{i}^{2}+z_{i}^{2}\right), I_{y}=\sum m_{i}\left(z_{i}^{2}+x_{i}^{2}\right), I_{z}=\sum m_{i}\left(x_{i}^{2}+y_{i}^{2}\right)$.

For continuously distributed masses the sums are replaced by integrals. For the moment of inertia about the $x$-axis we have:

$$
I_{x}=\iiint_{V} \sigma(x, y, z)\left(y^{2}+z^{2}\right) \mathrm{d} x \mathrm{~d} y \mathrm{~d} z .
$$

It is similar for the other axis.

The term axial moment of inertia can be applied both to masses spread across the surface and the curve, both in the case when the curve and axis lie in the same plane, and in the case when the curve, which can also be spatial, occupies an arbitrary position in relation to the axis of moment of inertia.

To better explain the afore mentioned mechanical phenomena, the concept of kinetic energy i.e. energy of movement can further be introduced. This highlights the importance of multiple integrals in mechanics. For a material point of mass $m$, moving at speed of intensity $v$, the kinetic energy is calculated by the form $\frac{1}{2} m v^{2}$.

When a solid body is moving translational its points have the same speed. Then taken for the representative of the body is the center of masses point $C$, and as the speed of all points $\overrightarrow{v_{c}}$ velocity is determined with intensity $v_{c}$. If $T$ marks the kinetic energy of a solid in the case of translational movement, then it follows:

$$
T=\frac{1}{2} \sum_{i=1}^{n} m_{i} v_{c}^{2}=\frac{1}{2} m v_{c}^{2}
$$

where $m$ is the overall mass of the body. Accordingly, in the translational motion, the kinetic energy of the body is expressed in the same way as the kinetic energy of a single material point. Now we look at a rotary motion of a solid body, i.e. rotation about a fixed axis. The velocity intensity $v_{i}$ of some point $M_{i}$ of the body which is at the distance $d_{i}$ from the axis can be calculated using the formula:

$$
v_{i} \lim _{\Delta t \rightarrow 0} \frac{\Delta S_{i}}{\Delta t}=\lim _{\Delta t \rightarrow 0} \frac{\mathrm{d}_{i} \Delta \alpha}{\Delta t}=\mathrm{d}_{i} \lim _{\Delta t \rightarrow 0} \frac{\Delta \alpha}{\Delta t}=\mathrm{d}_{i} \bar{\omega}
$$

where $\Delta S_{i}$ is the element of the roundabout of point $M_{i}, \Delta \alpha$ infinitely small rotation angle and:

$$
\bar{\omega}=\lim _{\Delta t \rightarrow 0} \frac{\Delta \alpha}{\Delta t}
$$

the intensity of angular velocity of the body. Thus, for the kinetic energy of each point of a solid body it can be put as follows:

$$
\frac{1}{2} m_{i} \mathrm{~d}_{i}^{2} \bar{\omega}^{2}=\frac{1}{2} \bar{\omega}^{2}\left(m_{i} \mathrm{~d}_{i}^{2}\right),
$$

and for the whole body we will get:

$$
T=\frac{1}{2} I \bar{\omega}^{2} \text { where } I=\lim _{n \rightarrow \infty} \sum_{i=1}^{n} m_{i} \mathrm{~d}_{i}^{2},
$$

i.e. moment of inertia of the body about the axis of rotation.

If we compare the expressions:

$$
T=\frac{1}{2} m v_{c}^{2} \text { and } T=\frac{1}{2} I \bar{\omega}^{2}
$$

we see that in the first expression the kinetic energy depends on the square of the linear velocity, and in the other on the square of the angular velocity. In the first expression we have a coefficient $m$, and in the second $I$ and both are called inertial coefficients-the first for the translational movement and the second for rotational movement.

\section{REFERENCES}

Awrejcewicz, J. 2012. In: Classical Mechanics, Springer, pp. 131185.

Bessonov, N. M. \& Song, D. J. 2001. Application of vector calculus to numerical simulation of continuum mechanics problems. Journal of Computational Physics, 167(1), pp. 22-38.

Bilimović, A. 1961. Integralni račun sa primenama.

Dorogovtsev, A. Y. 1987. Mathematical analysis: Collection of problems. Vishcha school.

Mirtich, B. 1996. Fast and accurate computation of polyhedral mass properties. Journal of graphics tools, 1(2), pp. 31-50.

Whitaker, S. 1992. The species mass jump condition at a singular surface. Chemical Engineering Science, 47(7), pp. 1677-1685. 